\title{
On the phase structure of a chiral invariant Higgs-Yukawa model
}

\section{Philipp Gerhold*}

Institut für Physik, Humboldt Universität zu Berlin, D-12489 Berlin, Germany

E-mail: gerholdephysik.hu-berlin.de

\section{Karl Jansen}

John von Neumann Institute for computing, NIC,

Platanenallee 6, D-15738 Zeuthen, Germany

E-mail: Karl.Jansen@desy.de

In the past the construction of Higgs-Yukawa models on the lattice was blocked by the lack of a consistent definition of a chiral invariant Yukawa coupling term. Here, we consider a chiral invariant Higgs-Yukawa model based on the overlap operator $\mathscr{D}^{\text {(ov) }}$ realized by the NeubergerDirac operator. As a first step towards a numerical examination of this model we study its phase diagram by means of an analytic $1 / N_{f}$-expansion, which is possible for small and for large values of the Yukawa coupling constant. In the case of strong Yukawa couplings the model effectively becomes an $O(4)$-symmetric non-linear $\sigma$-model.

XXIVth International Symposium on Lattice Field Theory

July 23-28, 2006

Tucson, Arizona, USA

\footnotetext{
*Speaker.
} 


\section{Introduction}

Non-perturbative investigations of Higgs-Yukawa models using the lattice regularization became subject of many investigations in the early 1990's $[1,2,3,4,5,6]$. These lattice studies were initially motivated by the interest in a better understanding of the fermion mass generation via the Higgs mechanism on a non-perturbative level and, in particular, in the determination of bounds on the Yukawa couplings translating into bounds on the Higgs boson mass and the - at that time not yet discovered - top quark mass. Furthermore, the fixed point structure of the theory received special attention due to the question whether besides the Gaussian also a non-trivial fixed point might exist. However, these investigations were blocked, since the influence of unwanted fermion doublers could not successfully be suppressed. Moreover, the models of these studies suffered the lack of chiral symmetry. The latter, however, would be indispensable for a consistent lattice regularization of chiral gauge theories like, for example, the standard model of electroweak interactions.

Here, we follow the proposition of Lüscher [7] for a chiral invariant lattice Higgs-Yukawa model based on the Neuberger overlap operator [8]. As a first step we begin with an analytical investigation of its phase structure by means of $1 / N_{f}$-expansions following $[9,10]$ and the references therein. We derive an expression for the effective potential at tree-level and present our preliminary results for the corresponding phase diagram.

In chapter 2 we briefly describe the considered model, before we present the large $N_{f}$ results at tree-level for small Yukawa couplings $y_{N} \propto 1 / \sqrt{N_{f}}$ in chapter 3. In the following chapter 4 we discuss a different large $N_{f}$-limit which becomes valid for non-vanishing Yukawa couplings. We then end with a short outlook.

\section{The model}

The chiral invariant Higgs-Yukawa model, which we consider here, contains one four-component, real Higgs field $\Phi$ and $N_{f}$ fermion doublets represented by eight-component spinors $\psi^{(i)}, \bar{\psi}^{(i)}$ with $i=1, \ldots, N_{f}$. Furthermore, there are also $N_{f}$ auxiliary fermionic doublets $\chi^{(i)}, \bar{\chi}^{(i)}$ only introduced to construct a chiral invariant Yukawa interaction term. The partition function can then be written as

$$
Z=\int D \Phi \prod_{i=1}^{N_{f}}\left[D \psi^{(i)} D \bar{\psi}^{(i)} D \chi^{(i)} D \bar{\chi}^{(i)}\right] \exp \left(-S_{\Phi}-S_{F}^{k i n}-S_{Y}\right)
$$

where the total action is decomposed into the Higgs action $S_{\Phi}$, the kinetic fermion action $S_{F}^{k i n}$, and the Yukawa coupling term $S_{Y}$. It should be stressed that no gauge fields are considered here.

The kinetic fermion action describes the propagation of the physical fermion fields $\psi^{(i)}, \bar{\psi}^{(i)}$ in the usual way according to

$$
S_{F}^{k i n}=\sum_{i=1}^{N_{f}} \sum_{n, m} \bar{\psi}_{n}^{(i)} \mathscr{D}_{n, m}^{(o v)} \psi_{m}^{(i)}-2 \rho \bar{\chi}_{n}^{(i)} \mathbb{1}_{n, m} \chi_{m}^{(i)}
$$

where the coordinates $n, m$ as well as all field variables and coupling constants are given in lattice units throughout this paper. The (doublet) Dirac operator $\mathscr{D}^{(o v)}=\hat{\mathscr{D}}^{(o v)} \otimes \hat{\mathscr{D}}^{(o v)}$ is given by the 
Neuberger overlap operator $\hat{\mathscr{D}}^{(o v)}$, which is related to the Wilson operator $\hat{\mathscr{D}}^{(W)}=\gamma_{\mu}^{E} \nabla_{\mu}-r \nabla_{\mu} \nabla_{\mu}$ by

$$
\hat{\mathscr{D}}^{(o v)}=\rho\left\{1+\frac{\hat{A}}{\sqrt{\hat{A}^{\dagger} \hat{A}}}\right\}, \quad \hat{A}=\hat{\mathscr{D}}^{(W)}-\rho, \quad \rho \geq 1
$$

where $\nabla_{\mu}$ denotes the symmetric difference quotient. It is well known that the eigenvalues $v^{ \pm}(p)$ of $\hat{\mathscr{D}}^{(o v)}$ with $\operatorname{Im}\left[v^{ \pm}(p)\right] \gtrless 0$ form a circle in the complex plane, the radius of which is given by the parameter $\rho$. In momentum space with $p \in \mathscr{P}=[-\pi, \pi]^{\otimes 4}$ these eigenvalues are explicitly given by

$$
v^{ \pm}(p)=\rho+\rho \cdot \frac{ \pm i \sqrt{\tilde{p}^{2}}+r \hat{p}^{2}-\rho}{\sqrt{\tilde{p}^{2}+\left(r \hat{p}^{2}-\rho\right)^{2}}}, \quad \tilde{p}_{\mu}=\sin \left(p_{\mu}\right), \quad \hat{p}_{\mu}=2 \sin \left(\frac{p_{\mu}}{2}\right) .
$$

The auxiliary fields $\chi^{(i)}$ on the other hand do not propagate. They do not have a direct physical interpretation at all and their contribution to the action is only introduced to establish chiral symmetry.

The Higgs field couples to the fermions according to the Yukawa coupling term

$$
S_{Y}=y_{N} \sum_{n, m} \sum_{i=1}^{N_{f}}\left(\bar{\psi}_{n}^{(i)}+\bar{\chi}_{n}^{(i)}\right) \underbrace{\left[\mathbb{1}_{n, m} \frac{\left(1-\gamma_{5}\right)}{2} \phi_{n}+\mathbb{1}_{n, m} \frac{\left(1+\gamma_{5}\right)}{2} \phi_{n}^{\dagger}\right]}_{B_{n, m}}\left(\psi_{m}^{(i)}+\chi_{m}^{(i)}\right)
$$

where $y_{N}$ denotes the Yukawa coupling constant and $B_{n, m}$ will be referred to as Yukawa coupling matrix. Here the Higgs field $\Phi_{n}$ is rewritten as a quaternionic, $2 \times 2$ matrix $\phi_{n}=\Phi_{n}^{0} \mathbb{1}-i \Phi_{n}^{j} \tau_{j}$ $\left(\tau_{j}\right.$ : Pauli matrices) acting on the flavor index. Due to the chiral character of this model, left- and right-handed fermions couple differently to the Higgs field, as can be seen from the appearance of the projectors $\left(1 \pm \gamma_{5}\right) / 2$ in the Yukawa term.

Finally, we use a slightly unusual notation for the Higgs action $S_{\Phi}$ given by

$$
S_{\Phi}=-\kappa_{N} \sum_{n, \mu} \Phi_{n}^{\dagger}\left[\Phi_{n+\hat{\mu}}+\Phi_{n-\hat{\mu}}\right]+\sum_{n} \Phi_{n}^{\dagger} \Phi_{n}+\lambda_{N} \sum_{n}\left(\Phi_{n}^{\dagger} \Phi_{n}-N_{f}\right)^{2}
$$

where $\kappa_{N}$ denotes the hopping parameter and $\lambda_{N}$ is the quartic coupling. The usual notation is reobtained by a trivial rescaling of the coupling constants and the Higgs field. The model then obeys an exact, but lattice modified chiral symmetry recovering the actual chiral symmetry in the continuum limit [7].

For the further analytical treatment of this model the fermionic degrees of freedom are integrated out leading to the effective action $S_{\text {eff }}[\Phi]$, which can be written in terms of fermionic determinants yielding

$$
S_{e f f}[\Phi]=S_{\Phi}[\Phi]-N_{f} \cdot \log \left[\operatorname{det}\left(y_{N} B \mathscr{D}^{(o v)}-2 \rho \mathscr{D}^{(o v)}-2 \rho y_{N} B\right)\right] .
$$

\section{Large $N_{f}$-limit for small $y_{N}$}

We now consider the limit of infinite fermion number $N_{f} \rightarrow \infty$ with the coupling constants scaling according to

$$
y_{N}=\frac{\tilde{y}_{N}}{\sqrt{N_{f}}}, \tilde{y}_{N}=\mathrm{const} \quad \lambda_{N}=\frac{\tilde{\lambda}_{N}}{N_{f}}, \tilde{\lambda}_{N}=\mathrm{const} \quad \kappa_{N}=\tilde{\kappa}_{N}, \tilde{\kappa}_{N}=\mathrm{const}
$$


which justifies a semi-classical approach to the investigated model. We will therefore directly evaluate the effective action (2.6), which is at least possible for the constant and the staggered mode of the Higgs field. Since the Higgs field scales proportional to $\sqrt{N_{f}}$ in the large $N_{f}$-limit, we apply the ansatz

$$
\Phi_{n}=\hat{\Phi} \cdot \sqrt{N_{f}} \cdot\left(m+s \cdot(-1)^{\sum_{\mu} n_{\mu}}\right)
$$

where $\hat{\Phi} \in \mathbb{R}^{4}$ with $|\hat{\Phi}|=1$ denotes a constant 4-dimensional unit vector. We will refer to $m, s$ as magnetization and staggered magnetization, respectively. For the actual evaluation of the effective action we rewrite (2.6), neglecting all constant terms independent of $\Phi$ in the following, as

$$
S_{e f f}[\Phi]=S_{\Phi}[\Phi]-N_{f} \cdot \log \left[\operatorname{det}\left(\mathbb{1}-\frac{y_{N}}{2 \rho} \cdot\left(\mathscr{D}^{(o v)}-2 \rho\right) \cdot\left[\mathscr{D}^{(o v)}\right]^{-1} \cdot B\right)\right] .
$$

For the effective potential $V_{\text {eff }}(m, s)$ at tree-level one then finally finds

$$
\begin{aligned}
\frac{1}{N_{f}} V_{e f f}(m, s) & =-\tilde{\kappa}_{N}\left(m^{2}-s^{2}\right)+m^{2}+s^{2}+\tilde{\lambda}_{N}\left(m^{4}+s^{4}+6 m^{2} s^{2}-2\left(m^{2}+s^{2}\right)\right) \\
& -\int_{p \in \mathscr{P}} \frac{d^{4} p}{(2 \pi)^{4}} \log \left[\left(1+\left(\frac{\tilde{y}_{N}}{2 \rho}\right)^{2}\left(m^{2}-s^{2}\right) \frac{|v(p)-2 \rho|}{|v(p)|} \cdot \frac{|v(\wp)-2 \rho|}{|v(\wp)|}\right)^{2}\right. \\
& \left.+m^{2}\left(\frac{\tilde{y}_{N}}{2 \rho}\right)^{2}\left(\frac{|v(p)-2 \rho|}{|v(p)|}-\frac{|v(\wp)-2 \rho|}{|v(\wp)|}\right)^{2}\right]
\end{aligned}
$$

with the abbreviations $\wp_{\mu}=p_{\mu}+\pi$.

The phase diagram can then be explored numerically by searching for the absolute minima of the effective potential $V_{e f f}(m, s)$ with respect to $m$ and $s$. In general, four types of solutions can be obtained. These are a symmetric (SYM: $m=0, s=0$ ), a ferromagnetic (FM: $m \neq 0, s=0$ ), an antiferromagnetic (AFM: $m=0, s \neq 0$ ), and a ferrimagnetic phase (FI: $m \neq 0, s \neq 0$ ). The corresponding phase diagram for the choice $\tilde{\lambda}_{N}=0.1$ is shown in Figure 1. Here, we only present a qualitative plot of the phase structure, since the obtained results are preliminary. However, the diagram reveals a rich phase structure. Especially, there is a ferrimagnetic phase, which was also observed in Monte Carlo studies of earlier, not chiral invariant Higgs-Yukawa models, for example in [11].

\section{Large $N_{f}$-limit for finite $y_{N}$}

We now consider a different limit of large fermion numbers where the coupling constants scale according to

$$
y_{N}=\tilde{y}_{N}, \tilde{y}_{N}=\mathrm{const} \quad \lambda_{N}=\frac{\tilde{\lambda}_{N}}{N_{f}}, \tilde{\lambda}_{N}=\mathrm{const} \quad \kappa_{N}=\frac{\tilde{\kappa}_{N}}{N_{f}}, \tilde{\kappa}_{N}=\text { const. }
$$

Again, the Higgs field scales proportional to $\sqrt{N_{f}}$ allowing to expand the effective action in powers of $1 /\left(y_{N}|\Phi|\right)$. In this large $N_{f}$-limit the power series expansion

$$
\begin{aligned}
S_{e f f}[\Phi] & =-N_{f} \cdot\left(\operatorname{Tr} \log [B]-\sum_{k=1}^{\infty} \frac{2^{k}}{k}\left(\frac{\rho}{y_{N}}\right)^{k} \operatorname{Tr}\left[\mathscr{D}^{(o v)}\left(\mathscr{D}^{(o v)}-2 \rho\right)^{-1} B^{-1}\right]^{k}\right) \\
& +S_{\Phi}[\Phi]
\end{aligned}
$$




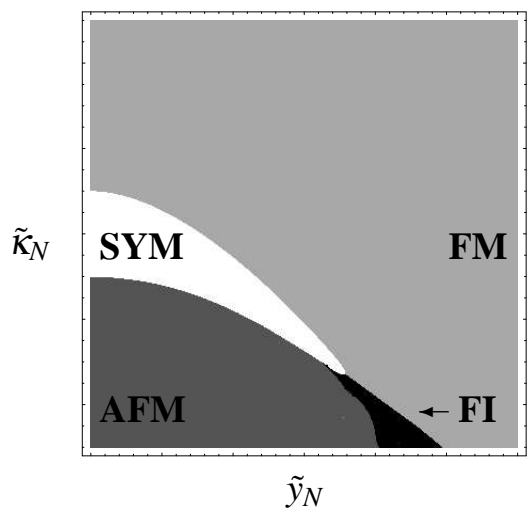

Figure 1: Qualitative phase diagram with respect to the Yukawa coupling $\tilde{y}_{N}$ and the hopping parameter $\tilde{\kappa}_{N}$ for a constant quartic coupling $\tilde{\lambda}_{N}=0.1$.

of the logarithm in equation (3.3) can therefore be cut off after the first non-vanishing term. The first summand $(k=1)$ is identical to zero and the second term $(k=2)$ is the first non-vanishing contribution. Cutting off the power series at $k=2$, the model then becomes an effective, $O(4)$ symmetric spin model

$$
S_{e f f}[\Phi]=S_{\Phi}[\Phi]-4 N_{f} \cdot\left(\sum_{n} \log \left(\left|\Phi_{n}\right|^{2}\right)+\frac{(2 \rho)^{2}}{y_{N}^{2}} \sum_{n, m} \frac{\Phi_{n}^{\mu}}{\left|\Phi_{n}\right|^{2}} \cdot K_{\mu v}(\Delta x) \cdot \frac{\Phi_{m}^{v}}{\left|\Phi_{m}\right|^{2}}\right)
$$

where $\Delta x=n-m$ and the non-local coupling matrix $K_{\mu v}(\Delta x)=\delta_{\mu, v} \cdot|\Gamma(\Delta x)|^{2}$ with $|$.$| denoting$ the 4-vector norm is explicitly given by the momentum integral

$$
\mathbb{R} \ni \Gamma_{\mu}(\Delta x)=\int_{p \in \mathscr{P}} \frac{d^{4} p}{(2 \pi)^{4}} e^{i p \Delta x} \cdot \frac{v^{+}(p)}{v^{+}(p)-2 \rho} \cdot \frac{\tilde{p}_{\mu}}{\sqrt{\tilde{p}^{2}}},
$$

which can be computed numerically. One then finds that the square-norm of the coupling matrix, $|K(\Delta x)|=|\Gamma(\Delta x)|^{2}$, decays exponentially with increasing distance $|\Delta x|$ as shown in Figure 2. In a field-theoretical sense the effective spin model therefore remains a locally interacting model.

For the evaluation of the corresponding phase diagram of this effective spin model we make the ansatz

$$
\Phi_{n}=\sqrt{N_{f}} \cdot \varphi_{0} \cdot \sigma_{n}, \quad \sigma_{n} \in \mathbb{R}^{4},\left|\sigma_{n}\right|=1, \quad \mathbb{R} \ni \varphi_{0}=\mathrm{const}
$$

for the Higgs field. Considering only the leading power in $1 / N_{f}$ of the tree-level effective action $S_{e f f}[\Phi]$, the amplitude $\varphi_{0}$ can be fixed according to

$$
0=-4 \cdot \frac{1}{\varphi_{0}^{2}}+1+2 \tilde{\lambda}_{N} \cdot\left(\varphi_{0}^{2}-1\right)
$$

Including the next to leading order terms in $1 / N_{f}$, the model effectively becomes an $O(4)$-symmetric non-linear $\sigma$-model

$$
\begin{aligned}
S_{e f f}[\Phi] & =-\sum_{n, m} \kappa_{n, m}^{e f f} \cdot \sigma_{n} \cdot \sigma_{m}, \quad \text { with } \\
\kappa_{n, m}^{e f f} & =\frac{16 \rho^{2}}{\tilde{y}_{N}^{2} \varphi_{0}^{2}} \cdot|\Gamma(\Delta x)|^{2}+\tilde{\kappa}_{N} \cdot \varphi_{0}^{2} \cdot \sum_{\mu= \pm 1}^{ \pm 4} \delta_{n, m+\hat{\mu}},
\end{aligned}
$$


the phase structure of which is again accessable to an $1 / N$-expansion. Here, $N$ denotes the number of components of the Higgs field $\Phi$, i.e. eventually $N=4$. As usual, the constraint $\left|\sigma_{n}\right|=1$ is removed by the introduction of an auxiliary field $\lambda_{n}$ according to

$$
\begin{aligned}
Z & =\int D \lambda \prod_{i=1}^{N}\left[D \sigma^{i}\right] \exp [-S[\sigma, \lambda]], \\
S[\sigma, \lambda] & =\frac{1}{t_{N}} \cdot\left\{\sum_{n, m} \sum_{i=1}^{N}-\kappa_{n, m}^{e f f} \cdot \sigma_{n}^{i} \cdot \sigma_{m}^{i}+\sum_{n} \lambda_{n} \cdot\left[\sum_{i=1}^{N}\left(\sigma_{n}^{i}\right)^{2}-1\right]\right\} .
\end{aligned}
$$

Integrating out the $N-1$ components $\sigma^{2}, \ldots, \sigma^{N}$ one obtains the reduced action $\hat{S}\left[\sigma^{1}, \lambda\right]$ depending only on the fields $\lambda_{n}$ and $\sigma_{n}^{1}$ given by

$$
\begin{aligned}
\hat{S}\left[\sigma^{1}, \lambda\right] & =-\sum_{n, m} \kappa_{n, m}^{e f f} \cdot \frac{1}{t_{N}} \cdot \sigma_{n}^{1} \cdot \sigma_{m}^{1}+\sum_{n} \lambda_{n} \cdot \frac{1}{t_{N}} \cdot\left[\left(\sigma_{n}^{1}\right)^{2}-1\right] \\
& +\frac{1}{2}(N-1) \operatorname{Tr}_{n, m} \log \left[-\kappa_{n, m}^{e f f}+\lambda_{n} \delta_{n, m}\right] .
\end{aligned}
$$

The newly introduced parameter $t_{N}$ is necessary to perform the large $N$-limit, which will be done for $\tilde{t}_{N} \equiv t_{N} \cdot N=$ const. Here, we are actually interested in the case $t_{N}=1$ and $N=4$. We therefore choose $\tilde{t}_{N}=4$. Again, we consider the magnetization and a possible staggered magnetization for the $\sigma_{n}^{1}$ field and assume the auxiliary $\lambda_{n}$ field to be constant leading to the ansatz

$$
\sigma_{n}^{1} \equiv m+s \cdot(-1)^{\sum_{\mu} n_{\mu}} \text { and } \lambda_{n} \equiv \lambda .
$$

Minimizing $\hat{S}\left[\sigma^{1}, \lambda\right]$ with respect to $m, s$, and $\lambda$ will then yield the three gap equations

$$
\begin{aligned}
0 & =m \cdot\left[\lambda-\left(8 \tilde{\kappa}_{N} \varphi_{0}^{2}+\frac{16 \rho^{2}}{\tilde{y}_{N}^{2} \varphi_{0}^{2}} \cdot q(0)\right)\right] \\
0 & =s \cdot\left[\lambda-\left(-8 \tilde{\kappa}_{N} \varphi_{0}^{2}+\frac{16 \rho^{2}}{\tilde{y}_{N}^{2} \varphi_{0}^{2}} \cdot q(\pi, \pi, \pi, \pi)\right)\right] \\
m^{2}+s^{2} & =1-\frac{\tilde{t_{N}}}{4}\left(1-\frac{1}{N}\right) \int_{k \in \mathscr{P}} \frac{d^{4} k}{(2 \pi)^{4}}\left[-\tilde{\kappa}_{N} \varphi_{0}^{2} \sum_{\mu} \cos \left(k_{\mu}\right)-\frac{8 \rho^{2}}{\tilde{y}_{N}^{2} \varphi_{0}^{2}} q(k)+\frac{\lambda}{2}\right]^{-1}
\end{aligned}
$$

where $q(k)$ denotes the eigenvalues of $|\Gamma(\Delta x)|^{2}$ corresponding to plane waves and is given by

$$
q(k)=\int_{p \in \mathscr{P}} \frac{d^{4} p}{(2 \pi)^{4}} \frac{v^{+}(p)}{v^{+}(p)-2 \rho} \cdot \frac{v^{+}(\wp)}{v^{+}(\wp)-2 \rho} \cdot \frac{\tilde{p} \cdot \tilde{\wp}}{\sqrt{\tilde{p}^{2}} \cdot \sqrt{\tilde{\wp}^{2}}}, \quad \wp=k-p .
$$

For the ferromagnetic phase, i.e. $m \neq 0$ and $s=0$, one obtains a self-consistent determination equation for $m^{2}>0$ according to

$$
0<m^{2}=1-\frac{\tilde{t_{N}}}{4}\left(1-\frac{1}{N}\right) \int_{k \in \mathscr{P}} \frac{d^{4} k}{(2 \pi)^{4}}\left[\tilde{\kappa}_{N} \varphi_{0}^{2} \sum_{\mu}\left(1-\cos \left(k_{\mu}\right)\right)+\frac{8 \rho^{2}}{\tilde{y}_{N}^{2} \varphi_{0}^{2}}(q(0)-q(k))\right]^{-1}
$$

An analogous relation holds for the antiferromagnetic phase (AFM). These relations can be treated numerically. The resulting phase diagram is shown in Figure 2. Again, we present a qualitative diagram only, since our results are preliminary. The appearance of three different phases, namely a symmetric, a ferromagnetic and an antiferromagnetic phase, can be observed. The presented phase diagram is in qualitative agreement with earlier results, using non-chiral fermions, see e.g. [9]. 

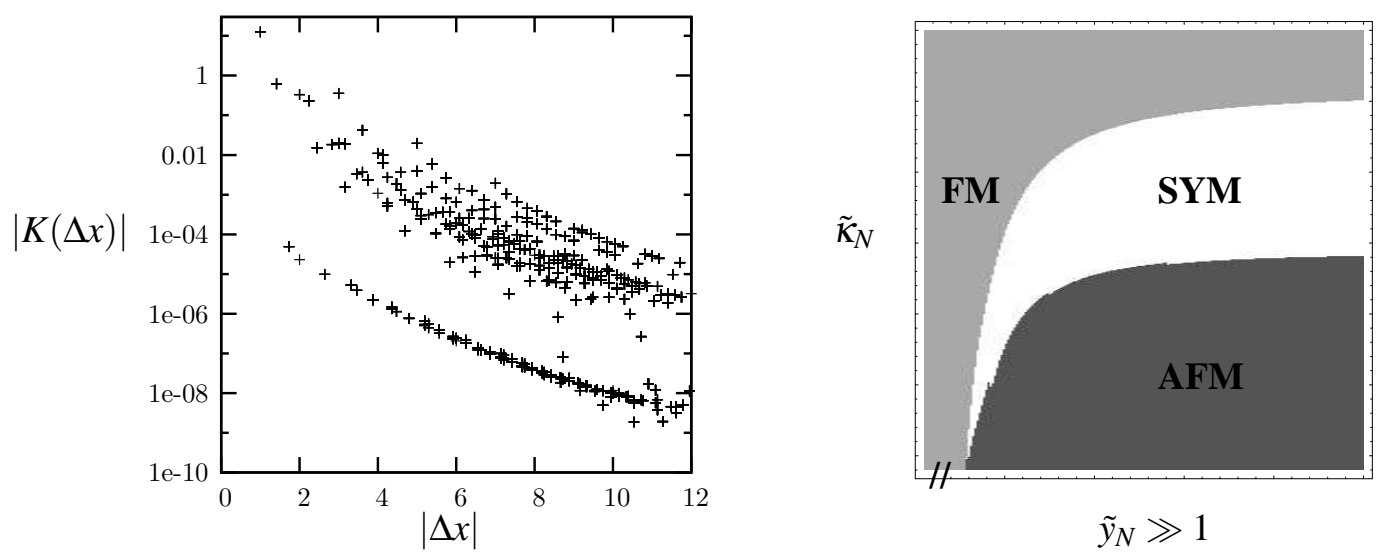

Figure 2: Left: Square-norm of the coupling matrix $K(\Delta x)$ versus the square-norm of the distance $\Delta x$ in lattice units. Right: Qualitative phase diagram with respect to the Yukawa coupling $\tilde{y}_{N} \gg 1$ and the hopping parameter $\tilde{\kappa}_{N}$ for a constant quartic coupling $\tilde{\lambda}_{N}=0.1$.

\section{Summary and outlook}

In this paper we have studied the phase structure of a chiral invariant lattice Higgs-Yukawa model, originally proposed by Lüscher, by means of analytic $1 / N_{f}$-expansions at tree-level. This was possible for small and for large values of the Yukawa coupling. Symmetric, ferromagnetic, and antiferromagnetic phases have been observed in both regimes of the Yukawa coupling constant. Additionally, a ferrimagnetic phase was found in the first regime. A next step would be the comparison of our analytical results with corresponding Monte Carlo data.

\section{Acknowledgements}

We thank the DFG for supporting this study by the DFG-project Mu932/4-1. Furthermore, we would like to thank Michael Müller-Preussker for helpful discussions and comments.

\section{References}

[1] J. Smit. Nucl. Phys. Proc. Suppl. 17, 3-16 (1990).

[2] J. Shigemitsu. Nucl. Phys. Proc. Suppl. 20, 515-527 (1991).

[3] M. F. L. Golterman. Nucl. Phys. Proc. Suppl. 20, 528-541 (1991).

[4] I. Montvay and G. Münster. Cambridge University Press (1997).

[5] A. K. De and J. Jersák. HLRZ Jülich, HLRZ 91-83, preprint edition (1991).

[6] M. F. L. Golterman, D. N. Petcher, and E. Rivas. Nucl. Phys. Proc. Suppl. 29BC, 193-199 (1992).

[7] M. Lüscher. Phys. Lett. B428, 342-345 (1998).

[8] H. Neuberger. Phys. Lett. B427, 353-355 (1998).

[9] A. Hasenfratz, P. Hasenfratz, K. Jansen, J. Kuti, and Y. Shen. Nucl. Phys. B365, 79-97 (1991).

[10] A. Hasenfratz, K. Jansen, and Y. Shen. Nucl. Phys. B394, 527-540 (1993).

[11] Wolfgang Bock et al. Nucl. Phys. B344, 207-237 (1990). 